\title{
Inter-annual variability influences the eco-evolutionary dynamics of range-shifting.
}

Understanding the eco-evolutionary dynamics of species under rapid climate change is vital for both accurate forecasting of biodiversity responses and for developing effective management strategies. Using an individual-based model we demonstrate that the presence and form (colour) of inter-annual variability in environmental conditions can impact the evolution of dispersal during range shifts. Under stable climate, temporal variability typically results in higher dispersal. However, at expanding margins, inter-annual variability actually inhibits the evolution of higher emigration propensities by disrupting the spatial sorting and natural selection processes. These results emphasize the need for future theoretical studies, as well as predictive modeling, to account for the potential impacts of inter-annual variability. 
1 Inter-annual variability influences the eco-evolutionary dynamics of range-shifting.

2 Roslyn C. Henry ${ }^{1 *}$, Greta Bocedi ${ }^{1}$, Calvin Dytham ${ }^{2}$ and Justin M.J. Travis ${ }^{1}$

3 1. Institute of Biological Sciences, University of Aberdeen, Zoology Building, Tillydrone

4 Avenue, Aberdeen, AB24 2TZ, UK.

5 2. Department of Biology, University of York, Heslington, York, YO10 5DD, UK

$6 \quad$ Author for correspondence: r01rch12@abdn.ac.uk

7 Running title: Dispersal, range shifting and inter-annual variability.

8 Keywords: Dispersal, Rangeshifter, climate change, environmental noise. 


\section{Summary}

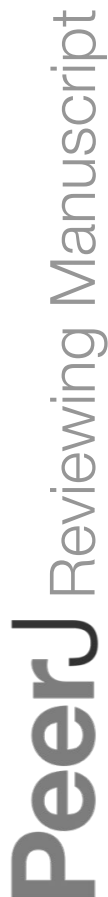

PeerJ reviewing PDF | (v2013:10:926:1:0:NEW 29 Nov 2013) 
10 Understanding the eco-evolutionary dynamics of species under rapid climate change is vital for

11 both accurate forecasting of biodiversity responses and for developing effective management

12 strategies. Using an individual-based model we demonstrate that the presence and form (colour)

13 of inter-annual variability in environmental conditions can impact the evolution of dispersal

14 during range shifts. Under stable climate, temporal variability typically results in higher dispersal.

15 However, at expanding margins, inter-annual variability actually inhibits the evolution of higher

16 emigration propensities by disrupting the spatial sorting and natural selection processes. These

17 results emphasize the need for future theoretical studies, as well as predictive modelling, to

18 account for the potential impacts of inter-annual variability. Introduction

19 Growing evidence suggests climate change is intensifying the inter-annual variability of the

20 climate and the frequency of extreme weather events, further increasing the temporal correlation

21 of unusual conditions (Coumou \& Rahmstorf 2012; Hansen et al. 2012). To survive global

22 warming species must either adapt in situ or shift their ranges to areas of newly suitable habitat

23 (Holt 2003; Parmesan 2006). Species have already exhibited distributional changes that are

24 consistent with those predicted by climate impact modelling (Chen et al. 2011). A species'

25 dispersal ability is central for determining its range shifting potential and is thus a key parameter

26 in predictive models. While in most models of range expansion individuals disperse with a

27 constant probability and/or move a distance drawn from a constant dispersal kernel (Kot et al.

28 1996), there is increasing evidence from both theoretical and empirical studies that dispersal will

29 come under strong and rapid selection during range shifts (Travis \& Dytham 2002; Hughes et al.

30 2007; B.L. Phillips et al. 2010; Kubisch et al. 2013). Although we understand how dispersal

31 should evolve in a stationary range with inter-annual variability in climate conditions (Kun \&

32 Scheuring 2006; Bocedi et al. 2012), theory on range shifting responses to climate change have 
33 typically only modelled changes in the mean rate of climate (Boeye et al. 2013; Henry et al.

34 2013). Thus most have not considered the additional effect of inter-annual variability, despite the 35 recognition that most natural systems exist in environments that are variable and positively

36 correlated (Inchausti \& Halley 2002; Vasseur \& Yodzis 2004).

37 In temporally constant and stable environments there is typically only limited selection

38 driving dispersal, e.g. pressure from kin selection (Ronce et al. 2000) and inbreeding avoidance

39 (Perrin \& Mazalov 1999; Perrin \& Mazalov 2000), and if emigrants suffer a cost by leaving their

40 natal patch, dispersal will remain low (Travis \& Dytham 1999; Dytham 2009). Environmental

41 variability, however, leads to greater uncertainty, increasing spatio-temporal variability of

42 environmental conditions and population density. In these situations a bet-hedging strategy,

43 dispersing to escape the possibility of local disaster, becomes advantageous (Friedenberg 2003).

44 Thus several previous studies have demonstrated selection for increased dispersal when

45 environmental variability is high and uncorrelated (Kun \& Scheuring 2006; Bocedi et al. 2012).

46 These results are from stationary ranges and the consequences of environmental variability for

47 dispersal evolution during range shifting remain unknown. Here, we develop some initial theory

48 to address this and show that environmental noise impedes the evolution of dispersal during

49 range shifting.

\section{Methods}

51 For the purpose of this study we use a spatially-explicit individual-based modelling platform,

52 RangeShifter (Bocedi et al. accepted). We model a haploid species with asexual reproduction and

53 discrete, non-overlapping generations on discrete random landscapes, where each individual is

54 characterized by its position on the landscape and dispersal characteristics. 


\section{Population Dynamics}

56 The landscape cells contain sub-populations of individuals, characterized by density-dependent

57 dynamics with demographic stochasticity. After dispersing, an individual settling in a suitable cell

58 within the climatic window (i.e. where $K>0$ ) reproduces. The dynamics of local populations are

59 simulated using an individual-based formulation of the Maynard Smith and Slatkin's (1973)

60 single species population model. In every generation $(t)$, each individual produces a number of

61 offspring randomly drawn from a Poisson distribution with mean $\mu_{t}$ given by the following

62 formula:

$$
\mu_{t}=\frac{r \cdot N_{t}}{1+(r-1) \cdot\left(\frac{N_{t}}{K}\right)^{b}}
$$

63 Where $r$ is the maximum growth rate at low densities, $b$ specifies the type of competition and

64 here was assumed to be equal to 1 ('contest' competition). $N_{t}$ is the number of individuals in a

65 cell at time $t$, and $K$ is the carrying capacity of the cell, both remain constant during offspring

66 production. This method of determining the number of offspring introduces demographic

67 stochasticity into the model. Individual emigration probabilities are initially randomly drawn

68 from a uniform distribution between 0 and 1. Offspring inherit the same emigration probability as

69 their parent with a probability of mutation $(m)$ equal to 0.001 . If a mutation occurs, the

70 emigration probability of the offspring assumes a random value drawn from a uniform

71 distribution between -0.1 and 0.1 the previous trait value. After reproduction all the adult

72 individuals die and the new populations consist of the offspring.

\section{Dispersal}


74 Emigration is density independent, at the start of each generation each new individual has a

75 probability of emigrating determined by its emigration trait value. The distance each individual

76 disperses is sampled from a negative exponential distribution with mean equal to 200 metres

77 (equal to two cells), while the direction is drawn from a uniform circular distribution (between 0

78 and $2 \pi$ radians). Individuals settle in a patch if it is suitable; if the patch is unsuitable the

79 individual dies. When all the dispersing individuals have either died or settled, the dispersal

80 phase is concluded and the model continues to the next generation step. Note that in using this

81 method not all individuals that have the opportunity to emigrate do effectively emigrate. A

82 proportion will draw a direction and distance resulting in them landing in their natal cell in which

83 case they do not emigrate. However, as the mean dispersal distance is constant, this does not lead

84 to confounding effects between evolution of emigration probability and dispersal distance.

\section{Landscape}

86 The simulations are performed on discrete random landscapes of 2000 by 50 cells with a single

87 habitat type. The resolution of each cell is 100 metres. Reflective boundary conditions are

88 applied. The landscape was randomly composed of suitable and unsuitable cells and for this

89 experiment $30 \%$ of the cells are randomly selected to be suitable. On top of this random

90 landscape an environmental gradient in cell carrying capacity $(K)$ is then applied linearly in space

91 along the $x$ axis. The gradient method follows that of Travis and Dytham (2004), optimal

92 conditions $\left(K_{\mathrm{opt}}=100\right)$ decline linearly to conditions that do not allow population survival $(K=0)$

93 resulting in a climate window of suitable habitat space. The carrying capacity of a suitable cell

94 with coordinates $x, y\left(K_{(x, y)}\right)$ is set by the following equation:

$$
K_{(x, y)}=K_{o p t}-\left|x-x_{o p t}\right| G
$$


96 Where $\left|x-x_{o p t}\right|$ is the distance from the cell to the optimum and $G$ is the gradient steepness

$97(G=2)$. Without environmental stochasticity this method results in a climate window that is 100

98 cells wide in the $x$ dimension of the landscape. $\mathrm{K}$ is therefore constrained to be greater than or

99 equal to zero.

100 To simulate a period of climate change the climatic window moves unidirectionally from left to 101 right (low to high values) along the $x$ axis at speeds varying from 0.125 to $2.0 x$ co-ordinates $t^{-1}$.

\section{Environmental noise}

103 To simulate inter-annual variability, a time series of $K$ modifiers is generated using a widely used

104 first-order autoregressive process detailed by Ruokolainen et al. (2009). Carrying capacity is

105 varied temporally according to the following equation:

$106 \quad K_{x, y, t}=K_{x, y, 0}+K \cdot \varepsilon_{t}$

107 Here, $K_{x, y, t}$ is the carrying capacity of the cell with coordinates $x$ and $y$ at time $t, K_{x, y, 0}$ is the 108 expected value of $K$ for the cell in absence of stochasticity and $K$ is the carrying capacity in 109 absence of gradient and stochasticity (i.e. $K_{\mathrm{opt}}$ ). $\mathcal{E}_{t}$ is the time series value determined by

$$
\varepsilon_{t}=\kappa\left(\varepsilon_{t-1}-1\right)+\omega_{t-1} \sqrt{1-\kappa^{2}}
$$

110 Where $\omega$ is a random normal variable sampled from $N(0, \sigma)$ and $\kappa$ is the autocorrelation. Here we 111 set standard deviation $(\sigma)$ to 0.25 . This equation produces positively correlated (red) noise when $112 \kappa$ is greater than zero and white noise when $\kappa$ is equal to zero. 


\section{Simulation experiments}

114 In each simulation, cells are initialised at carrying capacity and the optimum $\mathrm{x}$ co-ordinate is held

115 constant for 500 generations, shifted for 300, then stable for 500. Individual emigration

116 probability $(d)$ is randomly drawn from a uniform distribution between 0 and 1 . We run

117 simulations with no noise, uncorrelated (white) noise (standard deviation $\sigma=0.25$,

118 autocorrelation $\kappa=0.0)$ and positively correlated (red) noise $(\sigma=0.25, \kappa=0.9)$ to explore how

119 the presence and form of environmental variability influences the evolution of dispersal during 120 range expansion for 10 different rates of climate change (between 0 to $2 x$ co-ordinates $t^{-1}$ ).

\section{Results}

122 Prior to climate change the mean emigration probability is low in the core and increases towards 123 the range margins (Fig. 1a). The gradient in emigration probability becomes more pronounced 124 with environmental noise, with the highest emigration rates at the margins evolving under white 125 noise. Under red noise, populations are also surviving (at least some of the time) further from the 126 core. Consistently, across all scenarios during climate change (Fig.1b), the emigration probability

127 across the whole range increases but the pattern of the dispersal gradient across the range

128 changes, with low emigration at the rear of the range and higher emigration at the front. When

129 temporal environmental variability is present, at the rear of the range the evolved emigration rate

130 remains as before; white noise resulted in higher dispersal than red or no noise. However,

131 interestingly, at the expanding margin a switch occurs and scenarios with no noise evolve the 132 highest emigration rates. Apart from for the slowest rates of climate change we find the same 133 pattern, the mean emigration probability at the range front is higher in scenarios with no 
134 environmental noise (Fig. 2). The difference between the emigration probabilities evolving under

135 the different conditions decreases for the highest rates of climate change.

\section{Discussion}

137 Before climate change, emigration probability across the range evolves in response to both the 138 gradient structure and the nature of environmental noise. With a gradient in carrying capacity, 139 spatiotemporal variance in population dynamics increases towards the margin where $K$ is lower 140 and demographic stochasticity higher. This favours higher dispersal at the margins than in the

141 core due to bet hedging ( Ronce 2007; Kubisch et al. 2010). Thus, in all scenarios, we find higher

142 dispersal at the margins compared to the core. Environmental variability, particularly white noise, 143 no temporal autocorrelation, further increases the temporal variance and its effects are greatest at 144 the already fragile range margins. The gradient in emigration probability is therefore more

145 pronounced with substantially higher dispersal at the margins. Similar to Mustin et al. (2013), we

146 also find the range extent can become larger in positively correlated, red noise, scenarios as

147 consecutive generations of good conditions allow populations to colonize marginal habitats that

148 would otherwise remain unoccupied.

149 In this study our focus lies on the expanding margin however at this point it is also

150 important to highlight that there is considerable scope for work exploring the eco-evolutionary

151 dynamics at retreating margins. During climate change the emigration rate rapidly evolves to

152 higher levels at the front of the range. This evolutionary process is well documented (Hughes et

153 al. 2007; Phillips et al. 2010) and is the result of spatial sorting and natural selection (Shine et al.

154 2011). This increase in dispersal enables species to better track areas of suitable habitat as climate

155 change progresses, and may effectively act as an evolutionary rescue; without this process species 
156 might sometimes become extinct (Boeye et al. 2013; Henry et al. 2013). Furthermore,

157 irrespective of noise presence or colour, as the rate of climate change increased, the mean

158 emigration probability of individuals also increased. This is consistent with previous results that

159 higher rates of climate change drive the evolution of higher rates of dispersal (Boeye et al. 2013).

160 In all scenarios, at the end of climate change, the emigration probability is much lower at the

161 trailing edge of the range compared to the front and environmental noise typically increases

162 selection for higher emigration rates. However, at the front there is an interesting switch and now,

163 rather than temporal environmental variability increasing dispersal, it is in fact disrupting its

164 evolution. The environmental gradient results in poorer conditions (lower $K$ ) at the range front

165 compared with the core, these patches are therefore more susceptible to extinction in bad years.

166 Under temporal variability there is no longer a smooth advance of suitable environmental space,

167 instead populations will expand somewhat only to be knocked back following a run of poorer

168 years, this effect being more pronounced under positively autocorrelated variability. The most

169 dispersive individuals assorted into the furthest advanced patches in a period of better conditions

170 will be those prone to extinction when a sequence of poorer years occurs. This effectively

171 disrupts the spatial sorting and natural selection processes, reducing the evolved dispersal at the

172 front. Once climate change has ended the dispersiveness of individuals evolves back to levels

173 similar to those prior to climate change. Although our choice of landscape should not

174 quantitatively change the results, we highlight that it may influence the underlying evolutionary

175 processes after climate change. For example, with narrower climate windows the dispersiveness

176 of the whole population will increase rapidly as all individuals are forced to track a narrower area

177 of habitat and thus less dispersive genotypes will be lost. After climate change the decrease in

178 dispersiveness back to levels similar to those prior to climate change therefore requires the

179 evolution of lower dispersive genotypes to replace those lost in the range shifting process.

180 However with increasingly wider climate windows and invasion scenarios, where individuals are 
181 initialised at one end of the landscape and allowed to expand across empty space, habitat at the

182 rear of the range is less likely to disappear. Thus less dispersive genotypes at the rear of the range

183 are unlikely to be lost and once climate change has ceased the return to lower average dispersal

184 will not rely solely on new mutations but can be achieved by the spread of existing genotypes.

185 We suggest that similar effects of temporal environmental variability may occur for other life-

186 history traits that are likely to come under selection during range expansion (Burton et al. 2010;

187 Phillips et al. 2010). These effects may, in turn, disrupt the process of range shifting as failure to

188 evolve life history traits that promote range expansion may render species less able to track

189 shifting climate. Mustin et al. (2013) have already demonstrated that positively correlated

190 temporal variability increases the extinction risk in species during a period of climate change,

191 particularly when climate change is rapid. Mustin et al. (2013) did not include evolution in their

192 model and the impact of environmental variability is attributed to poor runs of environmental

193 conditions increasing patch extinction rates towards the front therefore reducing the pool of

194 patches from which the next wave of expansion can occur. Our results highlight another potential

195 mechanism whereby noise could increase extinction risk through the disruption of dispersal

196 evolution; in this case increasingly correlated environmental variability acts to constrain the

197 upwards evolution of dispersal at the expanding front thus reducing the potential for dispersal

198 evolution to rescue a species from climate change. We realise that the results presented here are

199 sensitive to mutation rate with the evolution of higher dispersal occurring faster with higher rates

200 of mutation and therefore the effect of interannual variability is reduced. However, the value used

201 in the results presented is already relatively high for such a simulation of a quantitative trait so we

202 believe the general effect is likely to robust for much realistic parameter space. Of course, future

203 modelling efforts should strive to establish a suite of models with explict and realistic genetic

204 architecture. 
Here, we have used a simple single species model and have explored scenarios where the

206 population dynamics are characterized by contest competition. It is well understood that as a

207 species' dynamics become more complex through over-compensatory density dependence,

208 dispersal is typically selected upwards (Holt \& Mcpeek 1996). However, it is not clear how these

209 inherent complexities of a population's dynamics will interact with environmental variability in

210 driving the eco-evolutionary dynamics of range expansion.

This is a topic worthy of future work. Additionally, some recent studies have begun to explore how dispersal evolves in communities (e.g. Schreiber and Saltzman 2009; Pillai et al.

213 2012; Travis et al. 2013) and there is concurrent interest in asking how communities will respond

214 to rapid climate change (Norberg et al. 2012; Urban et al. 2012; Singer et al. 2013; Phillips et al.

215 2010). Given that there is already good theoretical evidence that environmental noise can be

216 filtered in non-intuitive ways by the dynamics of interacting species (Ruokolainen et al. 2007;

217 Ranta et al. 2008), it will also be important to investigate the interplay between species

218 interaction and dispersal evolution during range shifts that occur in temporally variable 219 environments.

The nature of the population dynamics and the complexities of, for example, stage

221 structure, dispersal stages, environmental dependent transition rates, will almost certainly

222 influence how life histories evolve during expansions as a function of interannual variability. It is

223 beyond the scope of this paper to encompass all possible complexity. However our preliminary

224 study highlights the importance of inter-annual variability on climate induced range shifts and our

225 results emphasize that theoretical studies investigating the impact of climate change should

226 certainly consider the effects of environmental noise. Failure to do so risks reaching erroneous

227 conclusions about the evolution of dispersal and overestimation of species ability to tolerate a

228 period of climate change. This also holds true in an applied setting. European and North 
229 American systems are subject to decadal cycles of variability due to the Atlantic Multidecadal

230 Oscillation (Sutton \& Dong 2012) greatly influencing species demography. For example, a

231 drought lasting several years would impact upon recruitment and population persistence of many

232 plant species. However, most current projections of climate impact are based on trends in mean

233 climate and do not capture this potentially crucial effect. Future modelling, whether statistical or 234 process-based, should urgently seek to incorporate inter-annual variability.

\section{Acknowledgements}

236 Funding from the Natural Environment Research Council, UK (NERC) supported this research.

\section{Figure legends}

238 Fig.1. Mean emigration probability across the species range for different environmental noise

239 scenarios. (a) Prior to climate change, generation 500 and (b) following 300 generations of

240 climate change, generation 800. Black, grey and red points represent the scenarios with no, white

241 and red environmental noise respectively. The data shown are the averages of 2000 replicates for

242 each scenario, bars represent the standard error. Points with no standard error bars at the margins

243 of the range are generated from just one simulation run where a single simulation has produced a

244 slightly wider range than usual.

245 Fig.2. Mean and standard error of emigration probability of individuals in the front-most five

246 rows over the last 30 generations for different rates of climate change. Black, grey and red points

247 represent the scenarios with no, white and red environmental noise respectively. The data shown

248 are the averages of the first 100 replicates for each scenario. 


\section{References}

250

251

252

253

254

255

256

257

258

259

260

261

262

263

264

265

266

267

268

269

270

271

272

273

274

275

276

277

278

279

280

281

282

283

284

285
Bocedi, G., Heinonen, J. \& Travis, J M J, 2012. Uncertainty and the role of information acquisition in the evolution of context-dependent emigration. Am. Nat., 179(5), pp.606-620. Available at: http://www.ncbi.nlm.nih.gov/pubmed/22504543 [Accessed November 7, 2012].

Bocedi, G., Palmer, S.C.F., Pe’er, G., Heikkinen, R.K., Matsinos, Y.G., Watts, K. \& Travis, J.M.J. (accepted) RangeShifter: a platform for modelling spatial eco-evolutionary dynamics and species' responses to environmental changes.Methods in Ecology and Evolution.

Boeye, J., Travis, J.M.J, Stoks, R. \& Bonte, D. 2013. More rapid climate change promotes evolutionary rescue through selection for increased dispersal distance. Evol. Appl., 6(2), pp.353-364. Available at: http://doi.wiley.com/10.1111/eva.12004 [Accessed October 18, $2012]$.

Burton, O.J., Phillips, B. L. \& Travis, J.M.J, 2010. Trade-offs and the evolution of life-histories during range expansion. Ecol. Lett., 13(10), pp.1210-1220. Available at: http://www.ncbi.nlm.nih.gov/pubmed/20718846 [Accessed May 21, 2013].

Chen, I.C., Hill, J.K., Ohlemuller, R., Roy, D.B. \& Thomas C.D., 2011. Rapid range shifts of species associated with high levels of climate warming. Science, 333, pp.1024-1026.

Coumou, D. \& Rahmstorf, S., 2012. A decade of weather extremes. Nature Clim. Change, 2(March), pp.491-496. Available at: http://www.nature.com/nclimate/journal/v2/n7/full/nclimate1452.html [Accessed March 18, 2013].

Dytham, Calvin, 2009. Evolved dispersal strategies at range margins. Proc. R. Soc. B., 276(1661), pp.1407-1413. Available at:

http://www.pubmedcentral.nih.gov/articlerender.fcgi? artid=2677228\&tool=pmcentrez\&rendertype=abstract [Accessed March 13, 2012].

Friedenberg, N.A., 2003. Experimental evolution of dispersal in spatiotemporally variable microcosms. Ecol. Lett., 6(10), pp.953-959.

Hansen, J., Sato, M. \& Ruedy, R., 2012. Perception of climate change. PNAS, 109(37), pp.E2415-23. Available at: http://www.pubmedcentral.nih.gov/articlerender.fcgi? artid=3443154\&tool=pmcentrez\&rendertype=abstract [Accessed March 1, 2013].

Henry, R.C., Bocedi, G. \& Travis, J.M.J, 2013. Eco-evolutionary dynamics of range shifts: Elastic margins and critical thresholds. J. Theor. Biol., 321, pp.1-7. Available at: http://www.ncbi.nlm.nih.gov/pubmed/23246816 [Accessed March 17, 2013].

Holt, R.D., 2003. On the evolutionary ecology of species' ranges. Evol. Ecol. Res., 5, pp.159178.

Holt, R.D. \& Mcpeek, M.A., 1996. Chaotic population dynamics favors the evolution of dispersal. Am. Nat., 148(4), pp.709-718. 
286

287

288

289

290

291

292

293

294

295

296

297

298

299

300

301

302

303

304

305

306

307

308

309

310

311

312

313

314

315

316

317

318

319

320

321

322

323

324

325

326

327

328

329

330

331

332
Hughes, C.L., Dytham, Calvin \& Hill, Jane K., 2007. Modelling and analysing evolution of dispersal in populations at expanding range boundaries. Ecol. Entomol., 32(5), pp.437-445. Available at: http://doi.wiley.com/10.1111/j.1365-2311.2007.00890.x [Accessed March 17, 2013].

Inchausti, P. \& Halley, J., 2002. The temporal variability and spectral colour of animal populations. Evol. Ecol. Res., 4, pp.1033-1048.

Kot, M., Lewis, M.A. \& Driessche, P. Van Den, 1996. Dispersal data and the spread of invading organisms. Ecology, 77(7), pp.2027-2042.

Kubisch, A. et al., 2013. Kin competition as a major driving force for invasions. Am. Nat., 181(5), pp.700-706.

Kubisch, A., Hovestadt, T. \& Poethke, H.-J., 2010. On the elasticity of range limits during periods of expansion. Ecology, 91(10), pp.3094-3099. Available at: http://www.ncbi.nlm.nih.gov/pubmed/21058568.

Kun, Á. \& Scheuring, I., 2006. The evolution of density dependent dispersal in a noisy spatial population model. Oikos, 115(June), pp.308-320. Available at: http://onlinelibrary.wiley.com/doi/10.1111/j.2006.0030-1299.15061.x/full [Accessed March $17,2013]$.

Maynard Smith, J. \& Slatkin, M., 1973. The stability of predator-prey systems. Ecology, 54(2), pp.384-391.

Mustin, K., Dytham, C., Benton, T.G. \& Travis, J.M.J., (2013) Red noise increases extinction risk during rapid climate change. Diversity and Distrib. Available at: http://doi.wiley.com/10.1111/ddi.12038 [Accessed March 17, 2013].

Norberg, J., Urban, M.C., Vellend, M., Klausmeier, C.A. \& Loeuille, N., 2012. Eco-evolutionary responses of biodiversity to climate change. Nature Clim. Change, 2(8), pp.1-5. Available at: http://dx.doi.org/10.1038/nclimate1588.

Parmesan, C., 2006. Ecological and evolutionary responses to recent climate change. Annu. Rev. Ecol. Evol. Syst., 37(1), pp.637-669. Available at:

http://www.annualreviews.org/doi/abs/10.1146/annurev.ecolsys.37.091305.110100 [Accessed February 29, 2012].

Perrin, N. \& Mazalov, V., 1999. Dispersal and inbreeding avoidance. Am. Nat., 154, pp.282-292.

Perrin, N. \& Mazalov, V., 2000. Local competition, inbreeding, and the evolution of sex biased dispersal. Am. Nat., 155(1), pp.116-127.

Phillips, B L, Brown, G P \& Shine, R, 2010. Life-history evolution in range-shifting populations. Ecology, 91(6), pp.1617-1627.

Phillips, B.L., Brown, G.P. \& Shine, R., 2010. Evolutionarily accelerated invasions: the rate of dispersal evolves upwards during the range advance of cane toads. J. Evol. Biol., 23(12), pp.2595-2601. Available at: http://www.ncbi.nlm.nih.gov/pubmed/20939838 [Accessed March 1, 2013].

Phillips, B.L., Kelehear, C., Pizzatto, L., Brown, G.P., Barton, D. \& Shine, R., 2010. Parasites and pathogens lag behind their host during periods of host range advance. Ecology, 91(3), pp.872-881. Available at: http://www.ncbi.nlm.nih.gov/pubmed/20426344.

Pillai, P., Gonzalez, A. \& Loreau, M., 2012. Evolution of dispersal in a predator-prey metacommunity. Am. Nat., 179(2), pp.204-216.

Ranta, E. et al., 2008. Detecting compensatory dynamics in competitive communities under environmental forcing. Oikos, 117, pp.1907-1911.

Ronce, O., 2007. How does it feel to be like a rolling stone? Ten questions about dispersal evolution. Annu. Rev. Ecol. Evol. Syst., 38(1), pp.231-253. Available at: 

http://www.annualreviews.org/doi/abs/10.1146/annurev.ecolsys.38.091206.095611 [Accessed February 28, 2013].

Ronce, O., Gandon, S. \& Rousset, F., 2000. Kin selection and natal dispersal in an age-structured population. Theor. Popul. Biol., 58(2), pp.143-159. Available at: http://www.ncbi.nlm.nih.gov/pubmed/11042105.

Ruokolainen, L., Lindén, A., Kaitala, V. \& Fowler, M.S. 2009. Ecological and evolutionary dynamics under coloured environmental variation. Trends Ecol. Evol., 24(10), pp.555-563. Available at: http://www.ncbi.nlm.nih.gov/pubmed/19699550 [Accessed November 14, 2012].

Ruokolainen, L., Fowler, M.S. \& Ranta, E., 2007. Extinctions in competitive communities forced by coloured environmental variation. Oikos, 116, pp.439-448.

Schreiber, S.J. \& Saltzman, E., 2009. Evolution of predator and prey movement into sink habitats. Am. Nat., 174(1), pp.68-81.

Shine, Richard, Brown, G. \& Phillips, B., 2011. An evolutionary process that assembles phenotypes through space rather than through time. PNAS, 108(14), pp.8-11. Available at: http://www.pnas.org/content/108/14/5708.short [Accessed March 17, 2013].

Singer, A., Travis, Justin M J \& Johst, K., 2013. Interspecific interactions affect species and community responses to climate shifts. Oikos, 122, pp.358-366.

Sutton, R.T. \& Dong, B., 2012. Atlantic Ocean influence on a shift in European climate in the 1990s. Nat. Geosci., 5(11), pp.788-792. Available at: http://dx.doi.org/10.1038/ngeo 1595.

Travis, J M J et al., 2013. Evolution of predator dispersal in relation to spatio- temporal prey dynamics: How not to get stuck in the wrong place! PLoS one, 8(2), p.e54453.

Travis, J M J \& Dytham, C, 2002. Dispersal evolution during invasions. Evol. Ecol. Res., 4, pp.1119-1129. Available at: http://webpages.icav.up.pt/PTDC/BIABEC/098414/2008/Travis e Dytham 2002.pdf [Accessed March 22, 2013].

Travis, J M J \& Dytham, C, 1999. Habitat persistence, habitat availability and the evolution of dispersal. Proc. R. Soc. B., 266(1420), pp.723-728. Available at: http://rspb.royalsocietypublishing.org/cgi/doi/10.1098/rspb.1999.0696 [Accessed March 7, 2013].

Travis, J.M.J. \& Dytham, C., 2004. A method for simulating patterns of habitat availability at static and dynamic range margins. Oikos, 2, pp.410-416.

Urban, Mark C, Tewksbury, J.J. \& Sheldon, K.S., 2012. On a collision course : competition and dispersal differences create no-analogue communities and cause extinctions during climate change. Proc. R. Soc. B., 279, pp.2072-2080.

Vasseur, D.A. \& Yodzis, P., 2004. The color of environmental noise. Ecology, 85(4), pp.11461152. Available at: http://www.esajournals.org/doi/abs/10.1890/02-3122. 


\section{Figure 1}

\section{Figure 1}

Mean emigration probability across the species range for different environmental noise scenarios. (a) Prior to climate change, generation 500 and (b) following 300 generations of climate change, generation 800 . Black, grey and red points represent the scenarios with no, white and red environmental noise respectively. The data shown are the averages of 2000 replicates for each scenario, bars represent the standard error. Points with no standard error bars at the margins of the range are generated from just one simulation run where a single simulation has produced a slightly wider range than usual. 

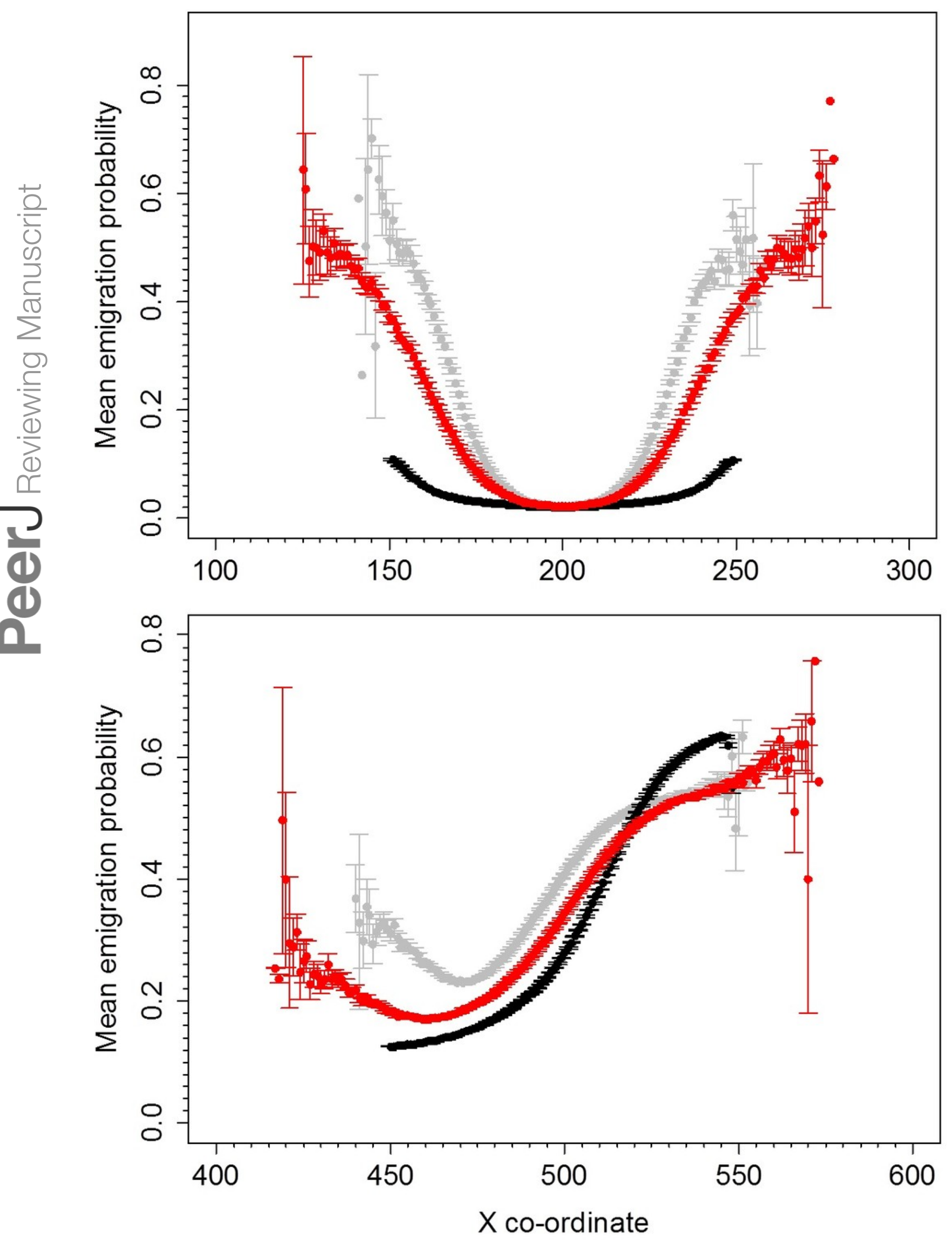


\section{Table 1 (on next page)}

Figure 2

Mean and standard error of emigration probability of individuals in the front-most five rows over the last 30 generations for different rates of climate change. Black, grey and red points represent the scenarios with no, white and red environmental noise respectively. The data shown are the averages of the first 100 replicates for each scenario. 


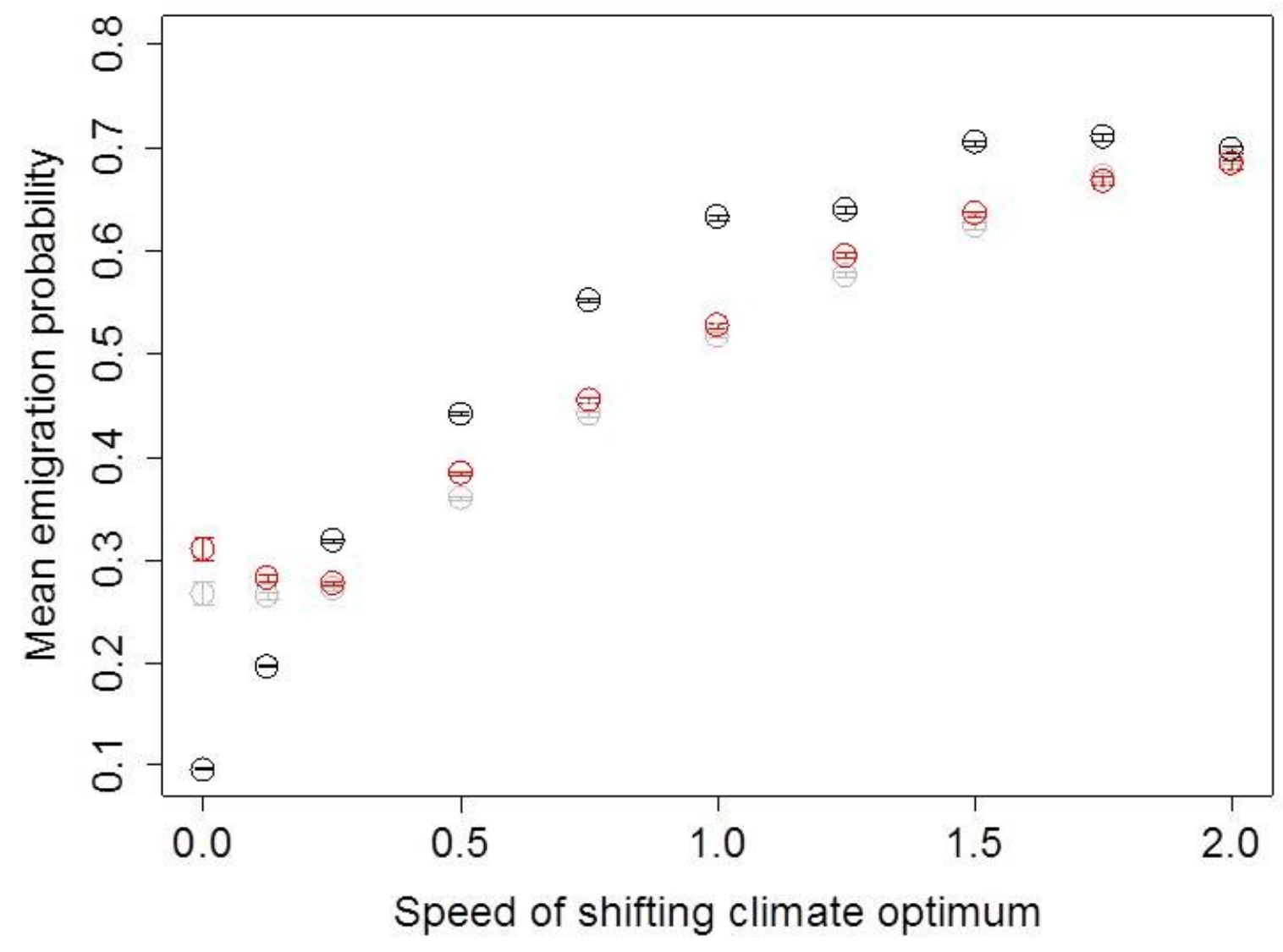

